\title{
nrDNA-ITS Variation, Structure, and Molecular Phylogeny of Abies beshanzuensis (Pinaceae), a Critically Endangered Species
}

\author{
Zengling Ma1,2, Yuping Sun ${ }^{1}$, Deliang Chen ${ }^{3}$, Changfeng Zhao', Qimeng Jiang', Qin Ke', Songjie \\ Pan $^{1}$, Xiaofeng Wang ${ }^{1,2^{*}}$
}

${ }^{1}$ College of Life and Environmental Science, Wenzhou University, Wenzhou 325035, China; mazengling@163.com (ZL, MA); sunyp508@126.com (YP, Sun); changfzhao@126.com (CF, Zhao); Jqm0577@163.com (QM, Jiang); keqwzu@126.com (Q, Ke); 37957861@qq.com (SJ, Pan)

${ }^{2}$ Zhejiang Provincial Key Laboratory of Subtropical Water Environment and Marine Biological Resources Protection, Wenzhou 325035, China

${ }^{3}$ Management Office of Beshanzu, Zhejiang Fengyang Mountain-Beshanzu National Nature Reserve, Qingyuan 323800, China; abiesqy@163.com (DL, Chen)

*Correspondence: alenixwang@126.com (XF, Wang); Tel.: +86 57786595039

\begin{abstract}
Abies beshanzuensis, an extremely endangered species, attracts serious concerns on genetic diversity recovery and population protection. However, little genetic information was known about it till now. In this study, intra-/intergenomic ITS variation, secondary structure, and molecular phylogeny of A.beshanzuensis were determined by nrDNA-ITS marker, and results indicated that, ITS region featured rich polymorphism in ITS1 and highly conservative in 5.8S/ITS2 among intra-/inter-genome, implying rich genetic diversities and the occurrence of non-concerted evolution in ITS region. Predicated 5.8S structure possessed Helices-Loops with 3 motifs (M1/M2/M3) and 5 helices B4-B8, and ITS2 could form conservative Clover-like structure with 5 ribotypes, manifesting genetically structural generalities and individualities. Thirteen ITS pseudogenes were also identified from 76 samples (or clones). Molecular phylogeny revealed that, A.beshanzuensis shared genetically closer relationships with A.fanjingshanensis, A.chensiensis, A.yuanbaoshanensis among genus Abies. The available original data provided rich genetic information on ITS sequence, structure and molecular phylogeny of A.beshanzuensis, which not only updated the databases of ITS sequences as well as secondary structures essential for phylogenetic reference and DNA barcoding analyses, but also contributed to deep exploration of population diversities, origin and evolution as well theoretical direction for population recovery and protection of A.beshanzuensis.
\end{abstract}

Keywords: Abies beshanzuensis; ITS variation; Structure; Molecular phylogeny

\section{Introduction}

As the second largest genus in Pinaceae, Abies is an ecologically significant for climate and economically important for timer. It comprises approximately 48 species, and discontinuously distributes from central and north America, eastern Asia to the Mediterranean area of Europe and 
southwestern Asia [1]. In China, approximately 28 Abies species (including varieties and imported species of A.firma), distribute from North to South China. Particularly, several rare, endangered species Abies beshanzuensis (Zhejiang), A. fanjingshanensis (Guizhou), A. yuanbaoshanensis (Guangxi) and A. ziyuanensis (Guangxi) have been attaching great attention from researchers and governments at all levels [2].

Abies beshanzuensis M.H.Wu (A. beshanzuensis), a critically endangered, endemic, ancient relic species, was putatively migrated from North to South at high altitude during Quaternary glacial period [3]. A. beshanzuensis only distributes in the extremely narrow Fengyang Mountain at the altitude of 1700 meters in Qingyuan county, China. Currently, only 4 naturally wild trees (one young and other 3 ancient) were discovered in their natural habitats, and approximately 2000 artificial trees were derived from these 3 ancient trees by direct seed breeding or xenostock grafting [4]. The extremely small population of individuals was possibly due to the following factors: 1) Extremely low hybridization rate due to imperfectly identical flowering period between male and female gametes; 2) Hybridization restriction from extremely limited individuals with island-isolated distribution in natural habitats; 3) Drastic interspecific survival competition pressure and fragile adaptation ability to ecological condition; 4) Possibly artificial destruction in the early or middle of the last century. However, the extremely small population of individuals would raise serious question of population diversities and recovery, ecological protection and sustainable development of A. beshanzuensis.

DNA molecular marker, played dominant roles in genetic diversities evaluation, phylogeny reconstruction, species identification and classification, and were successfully applied to Abies plants such as RFLP [5], AFLP and SSR [6], nrDNA-ITS [7] and/or combined/individual nuclear and organellar DNA [1,8-10] or nuclear microsatellites [11]. And these studies opened the door to deep exploring the origin and evolution, genetic diversities and phylogeny of genus Abies. Currently, an increasing data focused on the nrDNA-ITS analysis of genetic background, but there were still few reports on ITS variation and structure of Abies, particularly for A.beshanzuensis.

Eukaryotic nrDNAs regions, including 3 nrDNA genes (18S/5.8S/28S rDNA) and 2 internal transcribed spacers (ITS1/ITS2), as a complete transcription unit (18S-ITS1-5.8S-ITS2-28S), dispersed in genome hundreds of thousands of times [12]. ITS marker attracted serious concerns due to its rapid evolution and proper sizes for PCR-amplification as well as technological advantages of robustness and good repeatability [13]. So far, diverse ITS primers have been developed and widely applied for land plants [14,15]. Previous studies demonstrated big differences on sequences and lengths of ITS region (ITS1-5.8S-ITS2) among different family, genus or even species [16-19]. Compared with angiospermae, gymnosperms have much longer and more complex ITS sequence, particularly for Pinaceae plants $[7,17,19]$. Though thousands of ITS regions have been sequenced and deposited in NCBI database for many species, knowledges about their secondary structures were still limited [12,13,16,20,21-24]. To our knowledge, there were no reports on ITS sequence and structure from A.beshanzuensis till now.

rDNA-ITS variation of intraspecies were discovered in diverse species and high levels of intragenomic and intraspecific polymorphism attribute to formation of non-functional ITS (i.e. ITS pseudogenes) and of non-concerted evolution [25-27]. ITS secondary structures not only represented genetically structural information but also attributed to identification of ITS pseudogenes. The presence of ITS pseudogenes would cause the corresponding nrDNA pre-transcripts not to be 
correctly processed and maturated [12]. Thus, it's believed if ITS pseudogenes would be introduced into the phylogenetic analysis, it possibly produces erroneous hypothesis about phylogeny $[16,20,21,28]$. However, previous most studies on Pinaceae plants failed to care about the presence of ITS pseudogenes in phylogenies analysis.

The present study aimed to investigate nrDNA-ITS variation, structure, pseudogenes, non-concerted evolution and molecular phylogeny of A.beshanzuensis, which would contribute to deep exploration of genetic diversities, origin and evolution, biogeographical distribution as well as population recovery and protection of A.beshanzuensis.

\section{Results and Discussion}

\subsection{ITS sequence and length variation of A.beshanzuensis}

Great variation occurred on nucleotides sequence and length in ITS region in A.beshanzuensis (Table S1). The available data indicated that intra-/intergenomic ITS length ranged from 1411 (A.beshanzuensis A2-2) to 1755bp (A.beshanzuensis A4-2), in which ITS1 sizes ranged from 1006bp to $1350 \mathrm{bp}$, and the sizes of $5.8 \mathrm{~S}$ and ITS2 were $162 \mathrm{bp}$ and $243 \mathrm{bp}$ with some exceptions, respectively. The identities of ITS1/5.8S/ITS2 were $81.87 \% / 97.07 \% / 99.04 \%$, respectively, and average GC content in ITS1/ITS2 region (59.94\%/62.26\%) were remarkably higher than that in $5.8 S$ region $(50.93 \%)$. Apparently, variations on ITS sizes and nucleotides sequence for A.beshanzuensis mainly occurred in ITS1 region, which implied that ITS1, not 5.8S and ITS2, was responsible for most of variation of length and sequences. In contrast to ITS length of around 1700bp for most Abies species, longer ITS sizes were observed in A.grandis (2215bp, EF057690.1) (North America), A.concolor (2212bp, EF057692.1) (North America) and A.religiosa (2180bp, EF063714.1) (Central America). These species with longer ITS sizes featured highly similar morphological traits, and were regarded as evolutional Abies species [29]. Early data supported an "out of America" migration for the origin of an eastern Asian and western North America disjunct species pairs in section amabilis [1].

Subrepeats were present in ITS region in A.beshanzuensis. The available data supported 1-3 small subrepeats (SSR) (5'-GGCCACCCTAGTC-3') and the unique 298bp large subrepeats (LSR) in ITS1 region, implying these subrepeats largely contributed to ITS1 length variations among Abies species $[7,17]$. Previous reports displayed SSR sequences appeared in all Pinaceae while LSR sequences were only present in Abies [17] and in Picea [18]. Moreover, it's believed that, GC content in ITS1 region was relevant to its sequence length and subrepeats number [17]. Therefore, the sizes, GC contents, subrepeats and nucleotides variation of ITS regions provided basic genetic information for the origin and evolution of Abies plants [1].

\subsection{Non-concerted evolution analysis of A.beshanzuensis}

The polymorphism and lengths variations also appeared in intragenomic ITS region of A.beshanzuensis (Table 1 and Table S1). For 5 clones of A.beshanzuensis A1, their ITS identities were $94.61 \%$ with length variation from 1668 to $1717 \mathrm{bp}$, and nucleotides variation mainly occurred at sites 650-1085nt in ITS1 regions, including nucleotides indels and substitution (such as 6nt TTGGG insertion at sites 1038-1043nt in cloneA1-2 and A1-3) (Figure S1.1); For 4 clones in A.beshanzuensis A2, ITS identities of $88.97 \%$ was observed with nucleotides variations largely at sites $650-1247 \mathrm{nt}$ in ITS1 region (such as 4 fragments deletion of 30/81/21/120nt at different sites 838-1122nt in cloneA2-2 ) (Figure S1.2); Samples A.beshanzuensis A3 and A4 also shared similar variations on intragenomic ITS regions with $\mathrm{A} 1$ and $\mathrm{A} 2$, respectively, and their homologous identities from 5 clones were $89.72 \%$ and $93.77 \%$, respectively (Figure S1.3; Figure S1.4) (Table 1). Additionally, intragenomic ITS 
polymorphism were also observed in A.beshanzuensis D8, E21 etc as well as in A.fanjingshanensis in this study. Intra-/intergenomic ITS polymorphism implied the occurrence of non-concerted evolution in A. beshanzuensis and A.fanjingshanensis (Figure S1.5) (Table S1). Currently, an increasing data supported the occurrence of non-concerted ITS evolution in diverse species such as Lespedeza [25], Cycas [30], Pyrus [26], Mammillaria (Cactaceae) [23].

Table 1. Intragenomic ITS variation from 4 wild samples of A.beshanzuensis

\begin{tabular}{ccccccc}
\hline Sample & NC $^{*}$ & Size $(\mathrm{bp})$ & $(\mathrm{G}+\mathrm{C}) \%$ & Identity $(\%)$ & HVR $^{*}(\mathrm{bp})$ & Variance type \\
\hline A1 & 5 & $1633-1682$ & $59.17-60.00$ & 94.61 & $650-1085$ & Indel/substitution \\
A2 & 4 & $1376-1674$ & $56.54-59.86$ & 88.97 & $650-1247$ & Indel/substitution \\
A3 & 5 & $1383-1684$ & $58.28-59.99$ & 89.72 & $750-1250$ & Indel/substitution \\
A4 & 5 & $1643-1720$ & $59.24-60.01$ & 93.77 & $590-1163$ & Indel/substitution \\
\hline
\end{tabular}

Notes: NC* for number of clones; $\mathrm{HVR}^{*}$ for high nucleotides variance region.

\subsection{Secondary structure predication of $5.8 S$ and ITS2}

The 5.8S region of A.beshanzuensis could form typical "Helices-Loops" structure with 3 highly conserved motifs (M1/M2/M3) and helices (B4-B8) [21,31]. Of 3 motifs in all tested samples (Figure 1), M1 (CGATGAAGAACGTAGC) (16nt) participated in the formation of 2 helices of B4 and B5, M3 (UUUGAACGCA) (10nt) did the formation of another 2 helices of B4 and B7, while M2 (GAATTGCAGAATCC) (14nt) failed to participate in any helices, and only appeared in loop. All sequences of 3 conserved motifs in A.beshanzuensis were in accordance with those of Viridiplantae (M1: CGATGAAGAACGyAGC; M2: GAATTGCAGAAwyC; M3: TTTGAAyGCA) [21], which disclosed that the importance of these conservative motifs for $5.8 \mathrm{~S}$ structure and function in Viridiplantae. Differently, each species has base usage preference for motifs sites in $5.8 \mathrm{~S}$ structure.

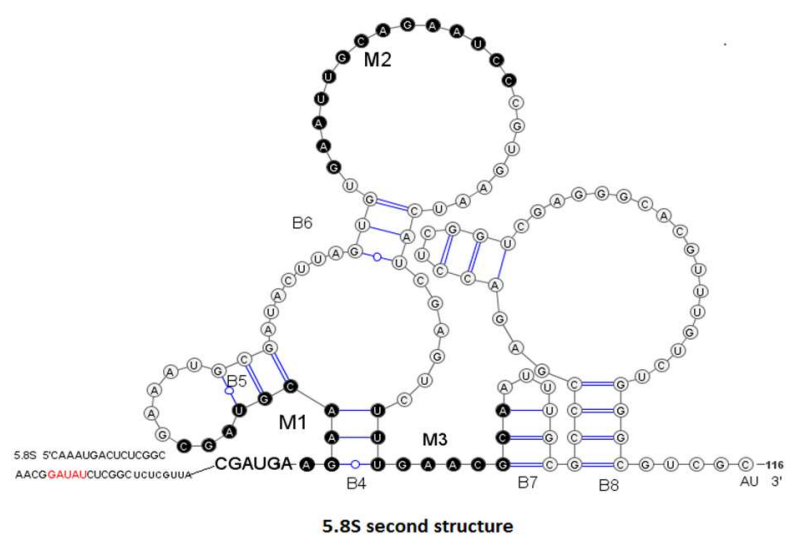

Figure 1. The secondary structure of $5.8 \mathrm{~S}$ consensus sequence (Notes: M1/M2/M3 for highly conservative motif; B4-B8 for helices) [31].

ITS2 regions of A.beshanzuensis (gymnosperm) could produce "Clover-like" structure with 4 helices (I/II/III/IV) radiating from one irregular/regular loop as well as non-canonical G-U base-pairs and UGGU motifs [16,24], and distinct differences were observed in each helices with different number, size and location of loops (Figure 2). Based on internal loop numbers in each helices, ITS2 structures from 35 sequences of A.beshanzuensis, were largely divided into 5 Ribotypes: Ribotype1 $(0,1,3,1)$, Ribotype2 $(0,1,4,0 / 1)$, Ribotype3 $(0,2,4,1)$, Ribotype4 $(0,3,4,1)$ and Ribotype5 $(2,2,3,0)$ (Figure 2). Briefly, Ribotype1 was present in samples A2-4 and A4-3, Ribotype2 occurred in sample A2-2 
$(0,1,4,0)$ and 29 samples $(0,1,4,1)$, and Ribotype3/4/5 only appeared in sample A3-3/A4-5/C8-2, respectively. Additional data disclosed A.chensiensis having Ribotype $(0 / 1,1,4 / 6,1 / 2)$ with one open loop between helices I and IV, and A.yuanbaoshanensis $(0 / 1,1,4 / 6,1)$ and A.fanjingshanensis $(1 / 2,1,2 / 6,1)$ with similar open loop at similar position as well as protruding loop on the left/right of helices III (Figure 2). This study supported slight differences of ITS2 structure with some exceptions in A.beshanzuensis (particularly in intra-individuals), and considerable differences among genus Abies. Generally, ITS2 structures represented evolutional and functional conservation as well as genetically structural diversities and uniqueness. Additionally, we failed to determine ITS1 structures of A.beshanzuensis, mainly due to that ITS1 regions had different number of shorter subrepeats (SSR)/longer subrepeat (LSR) sequences and high nucleotides variation rate, which would result in formation of extremely complex secondary structures [17], moreover, there lacked of effective methods to evaluate ITS1 structural differences.

Figure 2. Ribotypes for ITS2 structure of A.beshanzuensis and other Abies species in this study (Notes: I/II/III/IV for helices and the number in brackets for internal loop numbers in helices I/II/III/IV, respectively; L/R for protruding loop present in the left/right of helices III).

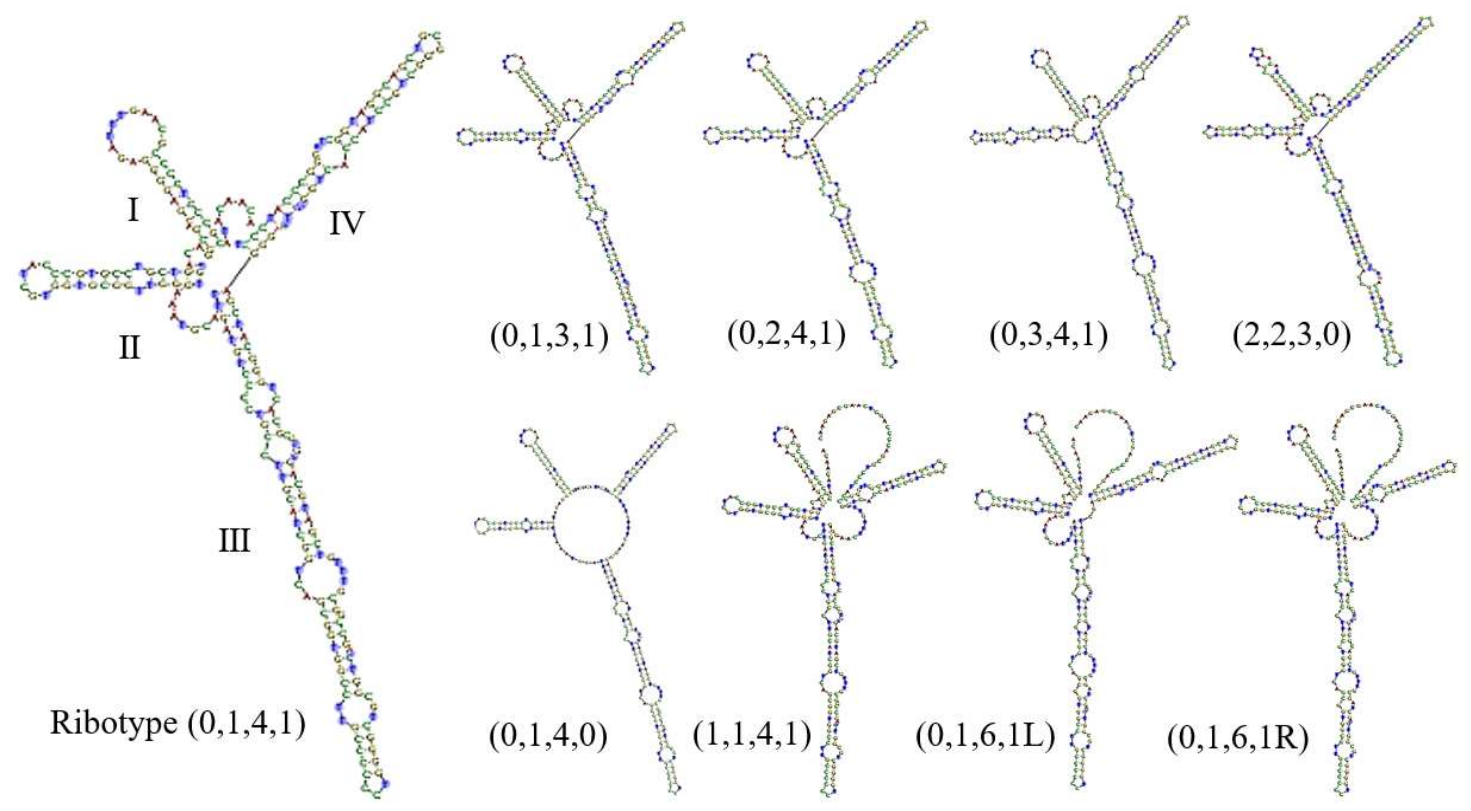

\subsection{Predication of ITS pseudogenes}

According to identification methods for ITS pseudogenes [21,22], nucleotides sequences in $5.8 \mathrm{~S}$ region were checked against the corresponding 3 motifs (M1: CGATGAAGAACGyAGC; M2: GAATTGCAGAAwyC; M3: TTTGAAyGCA) and 5 helices (B4-B8), the data disclosed that low level of mutations in M1 (16nt) for 4 samples: A2-2 (11th site of $C \rightarrow A$ ), A3-3 (12th G $\rightarrow$ A), C4-2 (16th C $\rightarrow$ $\mathrm{T}), \mathrm{C} 8-2$ (1st $\mathrm{C} \rightarrow \mathrm{T}$ and 8th $\mathrm{G} \rightarrow \mathrm{A})$, and that the 13th site of degenerate base was $\mathrm{T}$ not $\mathrm{C}$ in all samples. As for M3 (10nt), only single site variation (2nd T $\rightarrow$ C) was observed in sample A4-1, and the 7th site of degenerate base was $\mathrm{T}$ not $\mathrm{C}$ in all samples of A.beshanzuensis. Moreover, single nucleotide variation also appeared in sample A.spectabilis (2nd T $\rightarrow$ C) (JF416978.1). For M2 (14nt), all the samples had completely same sequences, and the degenerate bases WY in the 12th and 13th sites were $\mathrm{T}$ and $\mathrm{C}$, respectively, which implied that M2 possibly play core role in the formation of $5.8 \mathrm{~S}$ structure. In addition, several mutation sites were observed at non-motifs regions in 5.8S. Possibly, these mutations in highly conservative 5.8 motifs would result in ITS functional inactivation (ITS 
pseudogenes), and nucleotides variation in degenerate sites in $5.8 \mathrm{~S}$ motifs implied base usage preference for A.beshanzuensis plants. Based on combined factors of nucleotides variances in 5.8S, GC content, ITS size, and minimum free energy of 5.8S/ITS2 structures [21], 9 ITS pseudogenes were identified in 35 samples (or clones) of A.beshanzuensis and 4 ITS pseudogenes in other Abies species (Table S1). Totally, ITS pseudogenes featured nucleotides mutations in $5.8 \mathrm{~S}$ motifs, shorter ITS length, lower GC content, or unstable ITS2 structure, which contributed to extreme diversities and complex evolution of nrDNA [21,26,27].

\subsection{Phylogenetic reconstruction based on complete ITS1-5.8S-ITS2 sequence}

Phylogenetic tree of A.beshanzuensis was reconstructed based on complete ITS sequence (Figure 3). In this study, 13 identified ITS pseudogenes were excluded from 76 sequences. ITS sequences from 4 wild individuals of A.beshanzuensis were only considered for phylogeny of A.beshanzuensis, and single ITS sequence was selected from several clones of inter-/intra-individuals for each species $[1,7]$. The phylogenetic tree revealed that, 28 samples were distinctly divided into 2 clades: the major one having 25 samples with ITS sizes of approximately $1700 \mathrm{bp}$, and the minor one having 3 samples with ITS sizes of approximately 2200bp. Seemly, the ITS sizes played dominant role in phylogenetic analysis on Abies in this study.

In the major clade, A.fargesii (JF416972.1), endemic to central China, featured distinctively morphological traits and individually formed one clade. And the remaining 24 samples shared another clade including 2 Subclades I and II. Thirteen samples in Subclade I were originated from diverse areas such as East Asia (China, Japan, Korea) and Europe, while 11 samples in Subclade II formed 2 clusters with one having 5 samples native to western north America, and the other having 6 samples native to China. Seemly, weak correlation in Subclade I and strong correlation in Subclade II occurred between genetic similarities and geographical distribution.

Phylogenetic analysis represented sample A.beshanzuensis A1-1 was isolated from other 3 A.beshanzuensis samples (A2-1/A3-1/A4-2), suggesting sample A1 had genetically distant relationships with other 3 samples. Geographically, 3 wild ancient trees A1/A2/A3 clustered together and were approximately 50 meters away from sample A4. Moreover, all 4 wild individuals were in island-isolated distribution in their natural habitat. Thus, it was supposed that, the young tree A4, was possibly the hybrid offspring of ancient trees A1/A2/A3. This study supported A4 shared genetically close relationships with $\mathrm{A} 2$ and $\mathrm{A} 3$ and distant relationship with A1.

The phylogenetical relationships (Bootstrap:1000; Cutoff: 50\%) for 28 samples were well supported with exceptions of one cluster in Subclade I and 2 clusters in Subclade II. Previous studies proposed ITS marker was robust for phylogenetic analysis at interspecific or lower taxonomic levels [32], and the available data implied ITS marker was applicable for phylogenetic analysis of A.beshanzuensis. The present study implied that, A.beshanzuensis shared genetically closer relationships with A.fanjingshanensis, A.chensiensis, A.yuanbaoshanensis among genus Abies. Additionally, weak correlation between geographical distribution and genetic similarities appeared in several samples such as samples A.chensiensis a1 (Clades I) and A.chensiensis b1 (Clades II). The possible explanation was that, different ecological environments resulted in formation of varieties of A. chensiensis such as A. ernestii, A. ernestii var. salouenensis, A. recurvata [33], and introduction of Abies plants frequently occurred in both Nature Reserves, which possibly attributed to genetic variation for A.chensiensis a1 and b1. However, the true phylogenetic position of A.beshanzuensis as well as 
other species among genus Abies needed to be further valuated by data from diverse DNA markers, biochemical or morphological analysis.

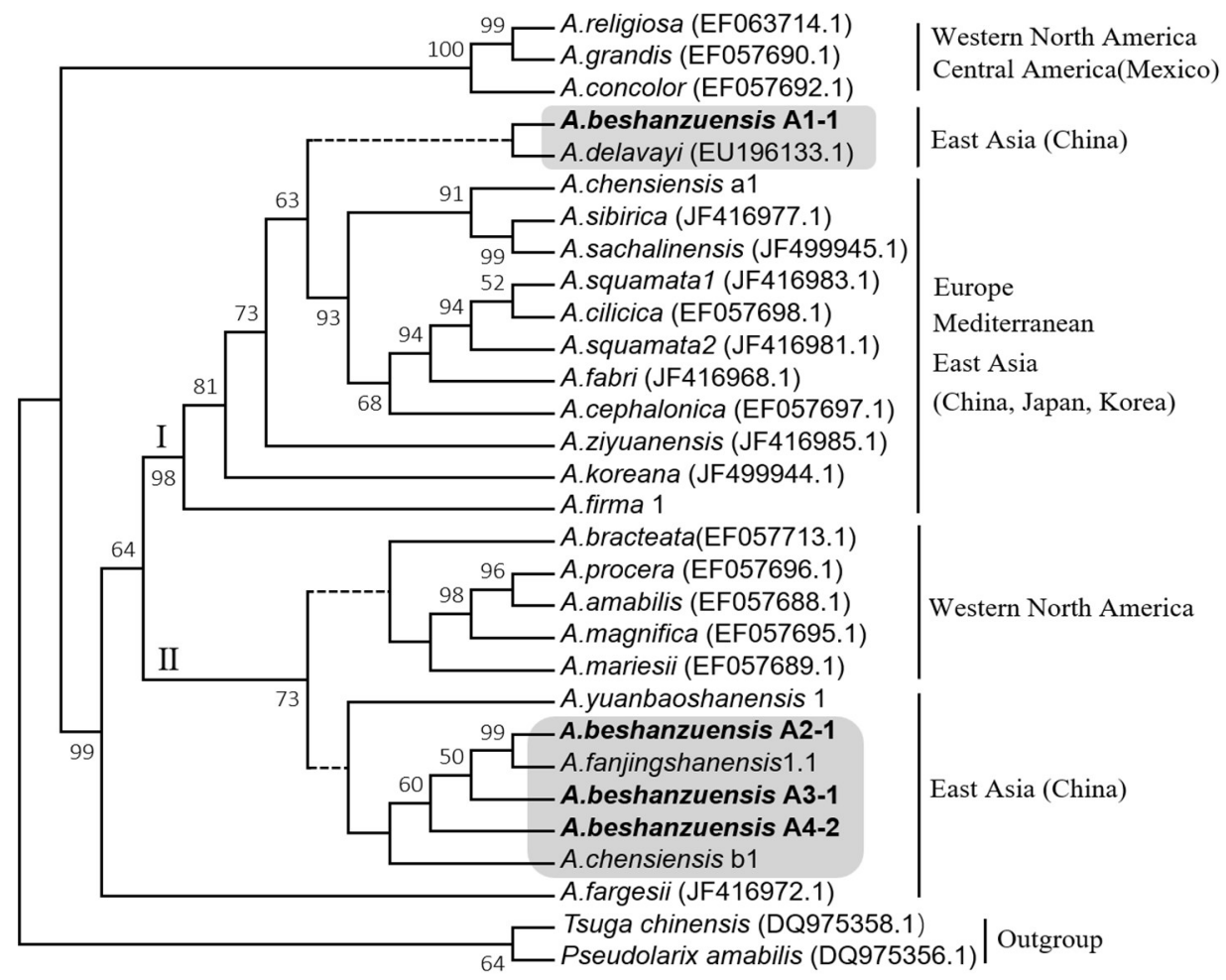

Figure 3. Phylogenetic tree inferred from ITS1-5.8S-ITS2 (Notes: N-J method and Kimura 2-parameter model, Bootstrap 1000; Cutoff $>50 \%$; Samples Tsuga chinensis (DQ975358.1) and Pseudolarix amabilis (DQ975356.1) for outgroups. The weakly-supported branches are indicated by broken line).

\section{Materials and Methods}

\subsection{Plant samples}

The young leaves from 24 samples were employed, including 13 individuals of A. beshanzuensis (4 wild trees and 9 artificial trees) and 11 other Abies plants in China (Table 2). All samples were completely cleaned by distilled water and stored in $-80^{\circ} \mathrm{C}$ freezer. Additionally, 26 ITS sequences from different Abies species were retrieved from NCBI database for comparative analysis (Table S1).

Table 2. Geographical distribution of A.beshanzuensis and other Abies plants in this study

\begin{tabular}{llllll}
\hline Abies species & Location & Size* $^{*}$ & Altitude/meter & Longitude & Latitude \\
\hline A.beshanzuensis & QingY, Zhejiang & $13 / 35$ & Approx.1700 & 119.2 & 27.6 \\
A.fanjingshanensis & TongR, Guizhou & $1 / 5$ & $2100-2300$ & 108.8 & 27.9 \\
A.yuanbaoshanensis & RongS, Gangxi & $3 / 3$ & $1700-2050$ & 109.1 & 25.4 \\
& LuS, Henan & $3 / 3$ & $2150-2800$ & 112.2 & 33.4 \\
A.chensiensis & LingB, Henan & $2 / 2$ & $2300-3000$ & 110.5 & 34.3 \\
A.firma (imported species) & WenZ, Zhejiang & $2 / 2$ & Approx.430 & 119.4 & 27.0 \\
Other Abies (NCBI) & Asia,America, & \multirow{2}{*}{$26 / 26$} & ---- & --- & - \\
& Europe & & & & - \\
\hline
\end{tabular}

Notes: * for samples number/clones number.

3.2. Genome DNA extraction and ITS fragment amplification 
Genomic DNA was extracted from tender leaves by modified CTAB method [50mM Tris.HCl (pH8.0), 10mM EDTA, 0.7mM NaCl, 1\%(W/V) CTAB, 20mM $\beta$-Mercaptoethanol)] [34], and one more purification was performed by organic solvent (Phenol/chloroform/isoamyl alcohol, 25:24:1) for completely removing proteins or other impurities. DNA concentration and quality were determined using a NanoDrop 2000 spectrophotometer (Thermo Scientific) and 1.0\% agarose gel electrophoresis visualized by ultraviolet (UV) light, respectively. And the final concentration of all genome DNA was diluted to $50 \mathrm{ng} / \mu \mathrm{l}$.

With the optimized reaction system and amplification program, genome DNA was amplified in $25 \mu \mathrm{l}$ cocktail including template DNA (50ng), $0.5 \mu \mathrm{l}$ forward primer (20mM) (ITS5: AGGAAGGAGAAGTCGTAACAAGG), $0.5 \mu \mathrm{l}$ reverse primer (20mM) (26S25R: TATGCTTAAACTCAGCGGGT) [19], 0.5 $\mu \mathrm{l}$ dNTP (10mM), 2.5 $\mu 1$ 10×PCR buffer, $0.5 \mu l$ DNA Taq (2U/ $\mu$ l, Dingguo, China) and appropriate volume of ddH2O; The PCR thermal cycler (Gene Amp PCR System 9600, Perkin Elmer, USA) was programmed by $5 \mathrm{~min}$ at $94^{\circ} \mathrm{C}$, followed by 35 cycles of $30 \mathrm{~s}$ at $94^{\circ} \mathrm{C}$ for denaturing, $30 \mathrm{~s}$ at $55^{\circ} \mathrm{C}$ for annealing, $110 \mathrm{~s}$ at $72^{\circ} \mathrm{C}$ for extension, and followed by another $10 \mathrm{~min}$ at $72^{\circ} \mathrm{C}$ for the final extension; PCR products were separated by $1.0 \%$ agarose gel electrophoresis.

\subsection{ITS fragment clone, sequence analysis and alignment}

Target DNA fragment (ITS1-5.8S-ITS2) was directly extracted from agarose gel based on the instruction of DNA gel extraction kit (Cat. DP209-02, Tiangen Biotech, China); Target DNA fragment was ligated into vector pMDTM18-T (Code: D101A, Takara, China) and transformed into competent cells from bacterial strain E.coli (DH5a) (Tiangen Biotech, China) according to manufacturer recommendations. Recombined plasmids were determined by colony PCR amplification from positive clones by primers ITS5/26S25R. And 4-5 identified clones from individual samples were randomly selected to investigate ITS polymorphism of intra-genome for some samples.

Target DNA sequence of recombined plasmid was analyzed by bidirectional sequencing using ABI genetic analyzer (ABI-PRISM3730XL) (Lifei biotechnology, China) with sequencing primers M13F (TGTAAAACGACGGCCAGT) and M13R (CAGGAAACAGCTATGACC) in vector pMDTM18-T, and sequences edition and alignment were conducted by software BioEdit (7.0), respectively. Based on sequence annotation of Abies plants deposited in NCBI database (GenBank: DQ975365.1; EU196130.1), the 5'-/3'- end boundaries of complete ITS1-5.8S-ITS2 region as well as respective ITS1, 5.8S, ITS2 regions were determined, respectively. Furthermore, the sequences and boundaries of 27 ITS retrieved from NCBI database were also performed for comparative analysis. ITS length, nucleotides variation, GC content and multiple sequence alignment were carried out by the softwares DNAstar and DNAMAN (8.0), respectively.

\subsection{Structure prediction, ITS pseudogene identification, phylogenetic reconstruction.}

$5.8 \mathrm{~S}$ secondary structure was predicted according to online software Mfold (RNA Folding Form) (http://unafold.rna.albany.edu/?q=mfold) and VARNA 3.9 (http://unafold.rna.albany.edu) with defaulted parameters (such as temperature and ionic condition etc.) [16]; ITS2 secondary structure and its minimum free energy were determined by direct folding or homologous modeling based on the online software with default setting, respectively (http://its2.bioapps.biozentrum.uni-wuerzburg.de) [24,35].

ITS pseudogenes determination mainly referred to the following factors: 1) Nucleotides variation on 3 motifs (M1/M2/M3) and helices B4-B8 in 5.8S structure; 2) GC content and length variation of 
ITS; 3) Minimum free energy of 5.8S and ITS2 structures [21,22]. Based on the complete sequence of ITS1-5.8S-ITS2, phylogenetic tree was reconstructed by Mega5.0 software with neighbor-joining method (NJ) and Kimura 2-parameter model (Cutoff $>50 \%$, Bootstrap 1000). Additionally, $5^{\prime}-/ 3^{\prime}$ - end boundaries-corrected complete ITS sequences from samples Tsuga chinensis (Pinaceae/Tsuga, DQ975358.1) and Pseudolarix amabilis (Pinaceae/Pseudolarix, DQ975356.1) were used as outgroups.

\section{Conclusions}

This study firstly reported intra-/intergenomic ITS sequence, structure, pseudogenes, non-concerted evolution, and molecular phylogeny of A.beshanzuensis (Pinaceae), a critically endangered species. The available data suggested that ITS region featured rich polymorphism with ITS1 region responsible for most of ITS variation, and that ITS pseudogenes and non-concerted evolution occurred during long-term evolution. Predicted structures of $5.8 \mathrm{~S}$ and ITS2 disclosed genetically structural information on universality and specificity. ITS phylogeny indicated A.beshanzuensis shared genetically closer relationships with A.fanjingshanensis, A.chensiensis, A.yuanbaoshanensis among Abies. This study would not only attribute to deep exploration of genetic diversities, origin and evolution, but also provide theoretical direction for hybrid breeding, species identification, population recovery and protection of A.beshanzuensis.

Supplementary Materials: Supplementary materials can be found at http://www.mdpi.com/xxxx; and the available ITS sequences in this study referred to GenBank MH177277-MH177291.

Author Contributions: Zengling Ma and Yuping Sun performed most of the experiments and data analyses; Deliang Chen provided some suggestion for research and participated in sample collection; Changfeng Zhao participated in experiments and data analysis; Qimeng Jiang and Qin Ke participated in data analyses; Songjie Pan participated in sample collection and preliminary experiments; Xiaofeng Wang conceived and designed the experiments and research, supervised the project and contributed to the writing of the manuscript. All authors read and approved the final manuscript.

Funding: This research was funded by Zhejiang Provincial Natural Science Foundation of China. Grant number [Code: LY13C060001].

Acknowledgments: We are thankful for supports by Zhejiang Fengyang Mountain-Baishanzu National Nature Reserve, Guangxi Yuanbao Mountain National Nature Reserve, Guizhou Fanjing Mountain National Nature Reserve, Henan Qinling Mountain National Nature Reserve (Lushan /Lingbao branch). And we also hope to thank professor Bingyang Ding (Wenzhou University, China) for his instruction.

Conflicts of Interest: The authors declare that there is no conflict of interest.

\section{Abbreviations}

nrDNA-ITS Nuclear Ribosomal DNA- internal transcribed spacer

RFLP Restriction fragment length polymorphism

AFLP Amplified fragment length polymorphism

SSR Simple-sequence repeat

PCR Polymerase chain reaction

NCBI The national center for biotechnology information

CTAB Cetyltrimethyl ammonium bromide

\section{References}

1. Xiang, Q.P.; Wei, R.; Shao, Y.Z; Yang, Z.Y.; Wang, X.Q.; Zhang, X.C. Phylogenetic relationships, possible ancient hybridization, and biogeographic history of Abies (Pinaceae) based on data from nuclear, plastid, and mitochondrial genomes. Mol. Phylogenet. Evol. 2015, 82,1-14. DOI: 10.1016/j.ympev.2014.10.008. 
2. Wang, Q.C.; Li, H.; Li, X.X. Geographical distribution characters and generating mechanisms of genus Abies in China. J. Cent. South. Univ. Forestry E Tech. 2012, 32 (9), 11-15 (In Chinese).

3. Kong, Z.C.; Du, N.Q.; Wu, M.X. A preliminary investigation of A. beshanzuensis: the present, past and future, Biodiversity and human future. China Forestry Publishing House. 1988, 141-144 (in Chinese).

4. Wu, Y.G.; Rao, L.B.; Chen, D.L.; Zhou, R.F.; Ye, Z.L. Artificial Seedling-raising of Abies beshanzuensis Seed. J.Anhui. Agri. Sci. 2010, 38(22), 12038-12039 (In Chinese).

5. Xiang, Q.P.; Xiang, Q.Y.; Liston, A.; Zhang, X.C. Phylogenetic relationships in Abies (Pinaceae): evidence from PCR-RFLP of the nuclear ribosomal DNA internal transcribed spacer region. Bot. J. Linn. Soc. 2004,145 (4), 425-435. DOI: org/10.1111/j.1095-8339.2004.00286.x.

6.Tang, S.; Dai, W.; Li, M.; Zhang, Y.; Geng, Y.; Wang, L.; Zhong, Y. Genetic diversity of relictual and endangered plant Abies ziyuanensis (Pinaceae) revealed by AFLP and SSR markers. Genetica. 2008, 133(1), 21-30. DOI: 10.1007/s10709-007-9178-x.

7. Xiang, Q.P.; Xiang, Q.Y.; Guo, Y.Y.; Zhang, X.C. Phylogeny of Abies (Pinaceae) inferred from nrITS sequence data. Taxon. 2009, 58(12), 141-152.

8.Aguirre-Planter, E.; Jaramillo-Correa, J.; Gómez-Acevedo, S.; Khasa, D.P.; Bousquet, J.; Eguiarte, L.E. Phylogeny, diversification rates and species boundaries of Mesoamerican firs (Abies, Pinaceae) in a genus-wide context. Mol. Phylogenet. Evol. 2012, 62(1), 263-274. DOI: 10.1016/j.ympev.2011.09.021.

9.Tollefsrud, M.M.; Sønstebø, J.H.; Brochmann, C.; Johnsen, Ø.; Skrøppa, T.; Vendramin, G.G. Combined analysis of nuclear and mitochondrial markers provided new insight into the genetic structure of North European Picea abies. Heredity. 2009, 102(6), 549-562. DOI: 10.1038/hdy.2009.16.

10.Suyama, Y.; Yoshimaru, H.; Tsumura, Y. Molecular phylogenetic position of Japanese Abies (Pinaceae) based on chloroplast DNA sequences. Mol. Phylogenet. Evol. 2000, 16(2), 271-277. DOI: 10.1006/mpev.2000.0795.

11.Awad, L.; Fady, B.; Khater, C.; Roig, A.; Cheddadi, R. Genetic structure and diversity of the endangered fir tree of Lebanon (Abies cilicica Carr.): implications for conservation. PLoS One. 2014, 9(2), e90086. DOI:10.1371/journal.pone.0090086.

12. Coleman, A.W. Nuclear rRNA transcript processing versus internal transcribed spacer secondary structure. Trends. Genet. 2015, 31, 157-163. DOI: org/10.1016/j.tig.2015.01.002.

13. Hřibová, E.; Čížková, J.; Christelová, P.; Taudien, S.; de Langhe, E.; Doležel, J. The ITS1-5.8S-ITS2 sequence region in the Musaceae: structure, diversity and use in molecular phylogeny. PLoS one. 2011, 6(3), e17863. DOI: 10.1371/journal.pone.0017863.

14. Cheng, T.; Xu, C.; Lei, L.; Li, C.; Zhang, Y.; Zhou, S. Barcoding the kingdom Plantae: new PCR primers for ITS regions of plants with improved universality and specificity. Mol.Ecol.Resour. 2016, 16(1), 138-149. DOI: 10.1111/1755-0998.12438.

15. Vijayan, K.; Tsou, CH. DNA barcoding in plants: taxonomy in a new perspective. Curr. Sci. 2010, 99(11), 1530-1541. URL: http://www.jstor.org/stable/24069450.

16. Rampersad, S.N. ITS1, 5.8S and ITS2 secondary structure modelling for intra-specific differentiation among species of the Colletotrichum gloeosporioides sensu lato species complex. SpringerPlus. 2014, 3, 684. DOI: 10.1186/2193-1801-3-684.

17. Kan, X.Z.; Wang, S.S.; Ding, X.; Wang, X.Q. Structural evolution of nrDNA ITS in Pinaceae and its phylogenetic implications. Mol. Phylogenet. Evol. 2007, 44(2), 765-777. DOI: 10.1016/j.ympev.2007.05.004.

18. Maggini, F.; Frediani, M.; Gelati, M. Nucleotide sequence of the internal transcribed spacers of ribosomal DNA in Picea abies Karst. DNA Seq. 2000, 11(1-2), 87-89. DOI: org/10.3109/10425170009033973. 
19. Liston, A.; Robinson,W.A.; Oliphant, J.M.; AlvarezBuylla, E.R. Length variation in the nuclear ribosomal DNA internal transcribed spacer region of nonflowering seed plants. Syst. Bot. 1996, 21, 109-120. DOI: $10.2307 / 2419742$.

20. Buchheim, M.A.; Keller, A.; Koetschan, C.; Förster, F.; Merget, B.; Wolf, M. Internal transcribed spacer 2 (nu ITS2 rRNA) sequence-structure phylogenetics: towards an automated reconstruction of the green Algal tree of life. PLoS One. 2011, 6(2), e16931. DOI: 10.1371/journal.pone.0016931.

21.Harpke, D.; Peterson, A. 5.8S motifs for the identification of pseudogenic ITS regions. Botany. 2008, 86(3), 300-308. DOI: org/10.1139/B07-134.

22.Harpke, D.; Peterso, A. Extensive 5.8S nrDNA polymorphism in Mammillaria (Cactaceae) with special reference to the identification of pseudogenic internal transcribed spacer regions. J. Plant. Res. 2008, 121(3), 261-270. DOI:10.1007/s10265-008-0156-x.

23.Harpke, D.; Peterson, A. Non-concerted ITS evolution in Mammillaria (Cactaceae). Mol. Phylogenet. Evol. 2006, 41(3), 579-593. DOI: 10.1016/j.ympev.2006.05.036.

24. Schultz, J.; Maisel, S.; Gerlach, D.; Müller, T.; Wolf, M. A common core of secondary structure of the internal transcribed spacer 2 (ITS2) throughout the Eukaryota. RNA. 2005, 11(4), 361-364. DOI:10.1261/rna.7204505.

25. Xu, B.; Zeng, X.M.; Gao, X.F.; Jin, D.P.; Zhang, L.B. ITS non-concerted evolution and rampant hybridization in the legume genus Lespedeza (Fabaceae). Sci. Rep. 2017, 7, 40057. DOI: 10.1038/srep40057.

26. Zheng, X.Y.; Cai, D.Y.; Yao, L.H.; Teng, Y.W. Non-concerted ITS evolution, early origin and phylogenetic utility of ITS pseudogenes in Pyrus. Mol. Phylogenet. Evol. 2008, 48(3), 892-903. DOI: 10.1016/j.ympev.2008.05.039. 27. Márquez, L.M.; Miller, D.J.; MacKenzie, J.B.; Van, Oppen. M.J. Pseudogenes contribute to the extreme diversity of nuclear ribosomal DNA in the Hard coral acropora. Mol. Biol. Evol. 2003, 20, 1077-1086. DOI:10.1093/molbev/msg122.

28. Poczai. P.; Hyvönen. J. Nuclear ribosomal spacer regions in plant phylogenetics: problems and prospects. Mol. Biol. Rep. 2010, 37(4),1897-1912. DOI: 10.1007/s11033-009-9630-3.

29. Xiang, Q.P.; Xiang, Q.Y.; Lison, A.; Fu, L.K.; Fu, D.Z. Length variation of the nuclear ribosomal DNA internal transcribed spacer in the genus Abies, with reference to its systematic utility in Pinaceae. Acta. Bot. Sin. 2000, 42, 946-951(In Chinese).

30.Xiao, L.Q.; Möller, M.; Zhu, H. High nrDNA ITS polymorphism in the ancient extant seed plant Cycas: incomplete concerted evolution and the origin of pseudogenes. Mol. Phylogenet. Evol. 2010, 55(1), 168-177. DOI: 10.1016/j.ympev.2009.11.020.

31.Wuyts, J.; De, Rijk. P.; Van, de.Peer. Y.; Winkelmans, T.; De, Wachter. R. The European large subunit ribosomal RNA satab20ase. Nucleic. Acids. Res. 2001, 29(1), 175-177. DOI: org/10.1093/nar/29.1.175.

32. Li, X.; Yang, Y.; Henry. R,J.; Rossetto, M.; Wang, Y.; Chen, S. Plant DNA barcoding: from gene to genome. Biol. Rev. Camb. Philos. Soc. 2015, 90(1):157-166. DOI: org/10.1111/brv.12104.

33. Shao, Y.Z.; Xiang, Q.P. Species delimitation and phylogeography of the Abies chensiensis complex inferred from morphological and molecular data. Bot. J. Linn. Soc. 2015, 177(2), 175-188. DOI: org/10.1111/boj.12235.

34.Zheng, W.H.; Zhuo, Y.; Liang, L.; Ding, W.Y.; Liang, L.Y.; Wang, X.F. Conservation and population genetic diversity of Curcuma wenyujin (Zingiberaceae), a multifunctional medicinal herb. Genet. Mol. Res. 2015, 14 (3), 10422-10432. DOI: 10.4238/2015.September.8.3.

35.Merget, B.; Koetschan, C.; Hackl, T.; Förster, F.; Dandekar, T.; Müller, T.; Schultz, J.; Wolf, M. The ITS2 Database. J. Vis. Exp. 2012, (61), 3806. DOI: 10.3791/3806. 